\title{
Nachhaltiges Informationsmanagement
}

DOI 10.1007/s11576-009-0188-4

\section{Die Autoren}

Dipl.-Wirt.-Inf. Nils-Holger Schmidt
Prof. Dr. Lutz M. Kolbe
Georg-August-Universität Göttingen
Professur für Informations-
management
Platz der Göttinger Sieben 5
37073 Göttingen
Deutschland
\{nschmid | lkolbe\}@
uni-goettingen.de

Dipl.-Ing. Koray Erek Prof. Dr. Rüdiger Zarnekow Technische Universität Berlin Lehrstuhl für Informations- und Kommunikationsmanagement Straße des 17. Juni 135 10623 Berlin

Deutschland \{koray.erek | ruediger. zarnekow\}@tu-berlin.de

Eingegangen: 2009-04-29 Angenommen: 2009-07-14 Angenommen nach einer Überarbeitung durch Prof. Dr. Sinz.

This article is also available in English via http://www.springerlink.com and http://www.bise-journal.org: Schmidt $\mathrm{N}-\mathrm{H}$, Erek K, Kolbe LM, Zarnekow R (2009) Sustainable Information Systems Management. Bus Inf Syst Eng. doi: 10.1007/s12599-009-0067-y.

\section{Die ökologische und soziale Dimension des Informationsmanagements}

Die zunehmende Verbreitung und steigende Abhängigkeit von Informationstechnologien (IT) und darauf aufsetzender Dienste führen zu bisher unberücksichtigten ökologischen und sozialen Herausforderungen. Das Informationsmanagement sieht sich aufgrund dieser Entwicklung mit neuen Anforderungen seitens der Geschäftsbereiche, der Kunden und der Mitarbeiter konfrontiert. Nachhaltiges Informationsmanagement erweitert die bislang primär ökonomisch orientierten Konzepte des Informationsmanagements um zwei neue Dimensionen, einer ökologischen und einer sozialen Perspektive.

In der Wissenschaft wird der ökologische Einfluss der IT auf Mensch und Umwelt in Form von Schadstoffen, Strahlung und Energieverbrauch schon seit vielen Jahren erforscht (Eder 1994, S. 600 ff). In der aktuellen Diskussion um „Green IT" steht der ökologische Einfluss der IT im Fokus öffentlicher und wissenschaftlicher Betrachtungen. IT-Dienstleister, wie beispielsweise Google, dessen 450000 Server pro Jahr 800 Gigawattstunden Elektrizität konsumieren, verantworten enorme Mengen indirekter $\mathrm{CO}_{2}$-Emissionen (Chou 2008, S. 93). Der Stromverbrauch aller Server weltweit entspricht in etwa dem Konsum der gesamten polnischen Volkswirtschaft (Koomey 2007).

Die Bedeutung sozialer Fragestellungen für das Informationsmanagement wird nicht zuletzt durch die in jüngster Zeit mit zunehmender Regelmäßigkeit in den Medien kolportierten Fälle von Datenmissbrauch deutlich, bei denen Unternehmen, Mitarbeiter oder Kunden betroffen sind. Der Umgang mit Daten innerhalb vieler Unternehmen wird als problematisch beschrieben (BSI 2009, S. 7). Infolgedessen rückt das Informationsmanagement verstärkt in das Blickfeld externer Interessensgruppen. Die soziale Dimension des nachhaltigen Informationsmanagements bezieht sich einerseits auf Themen der Anwendung von IT durch Individuen und Organisationen, andererseits fallen hierunter auch Herausforderungen der Generierung, Erhaltung und des Schutzes von Wissen, Informationen und Daten.

\section{Nachhaltigkeit und Ressourcenorientierung}

\subsection{Historie und Begriff der Nachhaltigkeit}

Das heute vorherrschende Verständnis der Nachhaltigkeit wurde durch die Definition der Brundtland-Kommission im Jahre 1987 geprägt, die „nachhaltige Entwicklung" als eine Entwicklung bezeichnet, die „den Bedürfnissen der heutigen Generation entspricht, ohne die Möglichkeiten künftiger Generationen zu gefährden, ihre eigenen Bedürfnisse zu befriedigen“" (Hauff 1987, S. 57).

Auf betrieblicher Ebene hat sich das Konzept der Nachhaltigkeit, ausgehend von Zielen der Ressourcenschonung und des Umweltschutzes, zu einer gleichberechtigten und gleichzeitigen Berücksichtigung ökonomischer, ökologischer und sozialer Ziele, dem Drei-SäulenModell der Nachhaltigkeit, weiter entwickelt. Nachhaltiges Management kann in diesem Zusammenhang als langfristiger, simultaner Optimierungsprozess von ökonomischen, ökologischen und sozialen Zielen zur Sicherung einer dauerhaften Geschäftstätigkeit definiert werden (Elkington 1997).

Das Drei-Säulen-Modell bietet Unternehmen und Organisationen einen Rahmen, ihr Wirken auf Wirtschaft, Gesellschaft und Umwelt zu strukturieren und zu beurteilen.

Adressaten eines nachhaltigen Managements sind insbesondere unternehmensinterne (z. B. Fachbereiche, Mitarbeiter) und -externe (z. B. Kunden, Eigentümer, Lieferanten, Geldgeber) Anspruchsgruppen, die über verschiedene Wirkmechanismen einen materiellen oder immateriellen Einfluss auf das Unternehmen ausüben und dadurch zu umwelt- und sozialverträglicher Wertschöpfung anreizen (Freeman 1984). Nachhaltiges Informationsmanagement spricht durch die ausgewogene Berücksichtigung ökonomischer, ökologischer und sozialer Aspekte die Bedürfnisse jeder dieser Anspruchsgruppen an. Dadurch erfolgt ein Beitrag zu den strategischen Zielen des Unternehmens und zu den erfolgskritischen Ressourcen des Informationsmanagements.

\subsection{Ressourcenorientierte Sicht auf das Informationsmanagement}

Im Kontext unternehmerischer Nachhaltigkeit hat sich die Ressourcentheorie als geeigneter Bezugsrahmen zur Schonung und Erhaltung strategisch wichtiger 


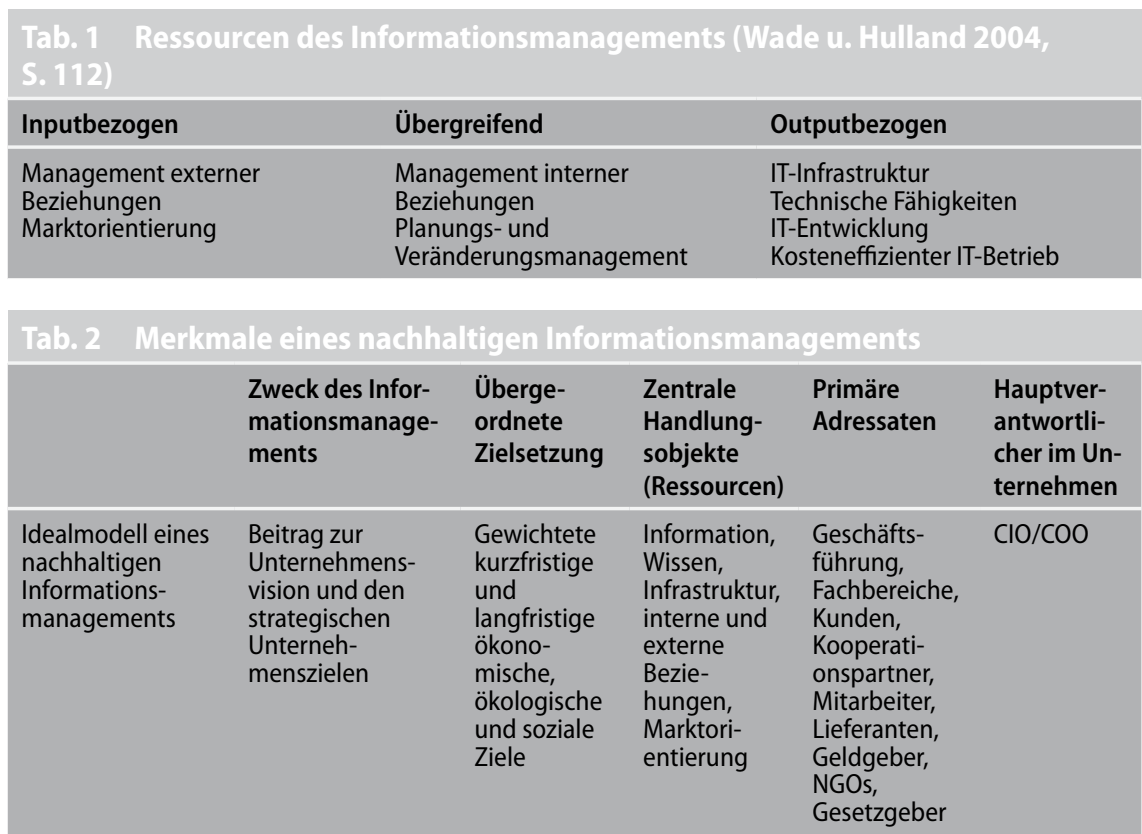

\begin{tabular}{|c|c|c|}
\hline $\begin{array}{l}\text { Prozessebene des Infor- } \\
\text { mationsmanagements }\end{array}$ & Soziale Zieldimension & Ökologische Zieldimension \\
\hline IT-Beschaffung & $\begin{array}{l}\text { Personalauswahl } \\
\text { Softwareauswahl } \\
\text { Lieferantenaudits } \\
\text { Verhandlung von SLAs }\end{array}$ & $\begin{array}{l}\text { Prüfung auf } \\
\text { Umweltzertifizierungen } \\
\text { Prüfung nach Öko-Labels } \\
\text { (z. B. Energy Star) } \\
\text { TCO-Analysen }\end{array}$ \\
\hline IT-Produktion & $\begin{array}{l}\text { Einhaltung von Datenschutz } \\
\text { und Datensicherheit } \\
\text { Wissensmanagement (z. B. Web 2.0) } \\
\text { Wissensgenerierung } \\
\text { Nutzung von Standards (z. B. ITIL) } \\
\text { Arbeitsplatzkonzepte (Shared Desk) } \\
\text { Kommunikationsmanagement } \\
\text { (z. B. Instant Messaging) }\end{array}$ & $\begin{array}{l}\text { Virtualisierungskonzepte } \\
\text { Grid-Computing } \\
\text { Cloud-Computing } \\
\text { Thin Clients } \\
\text { Kühlkonzepte im RZ } \\
\text { Wake-on-LAN } \\
\text { Einhaltung von Richtlinien } \\
\text { und Gesetzen }\end{array}$ \\
\hline IT-Vertrieb & $\begin{array}{l}\text { Sozialmarketing } \\
\text { Training der Kunden und Mitarbeiter } \\
\text { Dokumentation von Projekten } \\
\text { Beziehungsmanagement } \\
\text { zu Anspruchsgruppen }\end{array}$ & $\begin{array}{l}\text { Umweltmarketing } \\
\text { Erstellung von Umweltbilanzen } \\
\text { Beziehungsmanagement } \\
\text { zu Anspruchsgruppen }\end{array}$ \\
\hline
\end{tabular}

Unternehmensressourcen bewährt. Vor diesem Hintergrund liegt es nahe, die Frage aufzugreifen, welche Anstöße die Ressourcentheorie für die Entwicklung eines nachhaltigen Informationsmanagements leisten kann und wie diese effizient umgesetzt werden können. Die traditionelle, limitierte Fokussierung auf die Ressourcen Information, Kommunikation und Informationstechnik wird der heutigen Rolle des Informationsmanagements im Unternehmen nicht gerecht. Der Erfolg des Informationsmanagements ergibt sich aus Sicht der Ressourcentheorie aus dem Einsatz und der optimalen Kombination bestimmter materieller und immaterieller Ressourcen der Organisation. Wade u. Hulland (2004, S. 107 ff) identifizieren

\section{Aufbau eines nachhaltigen Informationsmanagements}

\subsection{Grundlagen und Merkmale}

Im deutschsprachigen Raum hat sich eine Vielzahl von Konzepten des Informationsmanagements etabliert. Grundsätzlich werden diese in problemorientierte, aufgabenorientierte, prozessorientierte, ebenenorientierte und architekturorientierte Konzepte unterschieden (Krcmar 2005, S. 28-47). Diese Konzepte beinhalten bereits viele Aspekte eines nachhaltigen Informationsmanagements, ohne sie jedoch explizit herauszustellen.

Anhand des vorgestellten Grundkonzepts der Nachhaltigkeit und der Ressourcentheorie kann ein idealtypisches Modell des nachhaltigen Informationsmanagements herausgearbeitet werden (Tab. 2).

Der Zweck eines nachhaltigen Informationsmanagements besteht in seinem Beitrag zur Unternehmensvision und -strategie sowie den daraus abgeleiteten strategischen Zielen des Unternehmens, in der neben ökonomischen auch ökologische und soziale Ziele konkret angesprochen werden.

Diese Ziele gelten nicht nur für die Ressource Information, sondern für alle, aus Sicht der Ressourcentheorie als wesentlich identifizierten, Ressourcen. Adressaten eines nachhaltigen Informationsmanagements sind explizit sämtliche Anspruchsgruppen. Da Umwelt- und Sozialthemen strategische Querschnittsaufgaben im Unternehmen sind, kann sich die Hauptverantwortung für das Informationsmanagement im Unternehmen auf die Ebene eines Chief Operating Officers (COO) verlagern.

ezüglich aus einer Vielzahl Studien acht wesentliche Kernressourcen des Informationsmanagements. Diese unterscheiden die Autoren in inputbezogene, übergreifende und outputbezogene Ressourcen (Tab. 1).

Die Ressourcentheorie geht von der Annahme aus, dass die Wettbewerbsvorteile und der damit einhergehende Erfolg einer Unternehmensfunktion, in diesem Fall des Informationsmanagements, davon abhängen, inwieweit es dazu beiträgt, diese Ressourcen zu beschaffen, effizient zu nutzen, zu erhalten und langfristig zu sichern (Wade u. Hulland 2004, S. $107 \mathrm{ff})$.

\subsection{Wirkungskreislauf} vorgestellten Einzelaspekte der Nachhaltigkeit, der Ressourcentheorie und des Informationsmanagements können im Rahmen eines Wirkungskreislaufs für nachhaltiges Informationsmanagement zusammengefasst werden (Abb. 1).

Im Mittelpunkt jeder planerischen Überlegung des nachhaltigen Informationsmanagements stehen die Bedürfnisse der Anspruchsgruppen (Abb. 1, (1.)). Je nach Bedeutsamkeit und Einflusspotential leiten sich gewichtete, kurz- und langfristige ökonomische, ökologische und soziale
Die in den vorangegangenen Abschnitten 
Ziele für das Informationsmanagement ab, die sich auf dem Drei-Säulen-Modell des nachhaltigen Managements begründen (Abb. 1, (2.)). Diese Ziele erreicht das Informationsmanagement durch Aufbau, Nutzung, Erhaltung und Sicherung seiner wesentlichen Ressourcen (Abb. 1, (3.)). Die dazu notwendigen Umsetzungsschritte erfolgen in Anlehnung an das Metamodell des Business Engineering auf der Strategie-, Prozess- und Systemebene des Informationsmanagements (Österle u. Blessing 2000) (Abb. 1, (4.)). Am Ende bedienen die konkreten Maßnahmen und Handlungsfelder des Informationsmanagements die Bedürfnisse der Anspruchsgruppen. Zahlreiche aktuell diskutierte Themen, wie z. B. Serviceorientierung oder GridComputing, lassen sich eindeutig durch die Ziele eines nachhaltigen Informationsmanagements motivieren (Abb. 1, (5.)).

Maßnahmen aus dem Bereich der „Green IT“ verbessern beispielsweise durch die Senkung des Energieverbrauchs die ökonomische und ökologische Bilanz (Buhl et al. 2009, S. 55). Dadurch werden Bedürfnisse der Geschäftsführung und der Fachbereiche, aber auch der Mitarbeiter und der Kunden, angesprochen. Die Ressourcen, die in diesem Zusammenhang eine wesentliche Rolle spielen, sind in erster Linie die Infrastruktur, die internen und externen Beziehungen sowie die Marktorientierung. Damit ist das Modell des nachhaltigen Informationsmanagements in der Lage, komplexe Beziehungen und Wirkungen zwischen Akteuren, Zielen, Ressourcen und Maßnahmen zu veranschaulichen.

\subsection{Maßnahmen und Umsetzung auf der Prozessebene}

Einzelne Maßnahmen können den Ebenen des Informationsmanagements zugeordnet werden. So betrifft beispielsweise der Einsatz von Servervirtualisierung im Rechenzentrum insbesondere den ITProduktionsprozess und die Systemebene, wohingegen komplexere Maßnahmen, wie unter anderem das Grid- oder CloudComputing, Anpassungen in der gesamten Strategie, den Prozessen und den Systemen des Informationsmanagements erforderlich machen.

Auf diese Weise lassen sich aktuelle Maßnahmen strukturiert einordnen. In Anlehnung an Schmidt et. al. (2009) kann beispielsweise die Prozessebene des nachhaltigen Informationsmanagements

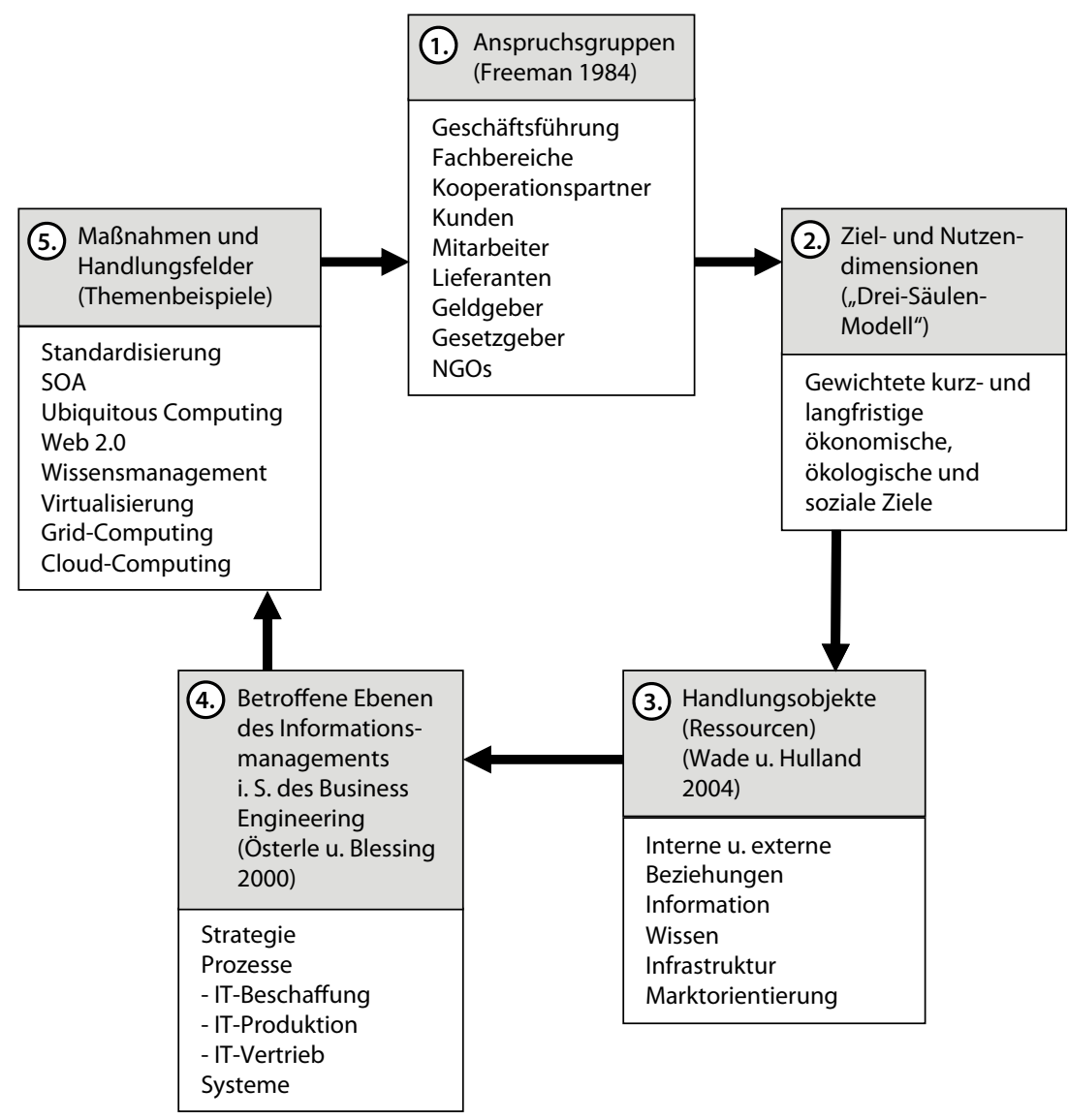

Abb. 1 Wirkungskreislauf eines nachhaltigen Informationsmanagements

betrachtet und mit der ökologischen und sozialen Zieldimension in einem Portfolio verknüpft werden. Dadurch ergeben sich vielfältige Kombinationsmöglichkeiten (Tab. 3). Auf diese Weise kann gezeigt werden, welchen - über die ökonomische Dimension hinausgehenden Einfluss eine Maßnahme haben kann.

\section{Bedeutung für die Wirtschaftsinformatik}

In Zukunft wird die Bedeutung ökologischer und sozialer Themen im Informationsmanagement weiter zunehmen. Einerseits soll sich der durch die IT verursachte Anteil an den gesamten $\mathrm{CO}_{2}$-Emissionen bis zum Jahr 2020 auf drei Prozent erhöhen. Andererseits kann die IT durch intelligente Geschäfts- und Produktionsprozesse oder durch Umweltinformationssysteme einen wichtigen Beitrag zur Ressourcenschonung im Unternehmen leisten (Buhl et al. 2009, $54 \mathrm{ff}$ ). Diese nach innen und außen gerichteten Perspektiven werden im Konzept des nachhaltigen Informationsmanagements integriert.

Für die Wirtschaftsinformatik bietet sich durch die explizite Berücksichtigung der Anspruchsgruppen, der Nachhaltigkeitsziele und der Ressourcen ein ganzheitlicher Strukturierungsansatz mit einer Vielzahl von Forschungsfragen. So stellt sich die Frage, durch welche konkreten Maßnahmen die IT dazu beitragen kann, die spezifischen ökologischen und sozialen Bedürfnisse einzelner externer und interner Gruppen zu befriedigen. In diesem Zusammenhang sind insbesondere die Potentiale und Risiken der zunehmenden Vernetzung zu erkunden und Implikationen für das Informationsmanagement in den Unternehmen abzuleiten.

Entwickelt sich die IT analog zu anderen Branchen, z. B. der Automobilindustrie, dann bleibt zu erwarten, dass Umwelt- und Sozialthemen sowie regulatorische Rahmenbedingungen zukünftig eine größere Rolle spielen werden. So geben in zahlreichen Großunternehmen die Geschäftsbereiche der IT quantita- 
tive Nachhaltigkeitsziele vor. Die Ergänzung bestehender Ansätze um eine ökologische und soziale Dimension erscheint daher sinnvoll.

Die Wirtschaftsinformatik kann zukünftig mit einer stärkeren Integration sozialwissenschaftlicher Perspektiven, im Zusammenspiel mit einer Renaissance der Ressourcentheorie, ein breites, gesellschaftlich relevantes Forschungsfeld besetzen und zu dessen systematischer Erforschung einen wichtigen Beitrag leisten.

\section{Literatur}

Buhl HU, Laartz J, Löffler M, Röglinger M (2009) Green IT reicht nicht aus!. Wirtschaftsinformatik \& Management 1(1):54-58

BSI - Bundesamt für Sicherheit in der Informationstechnik (2009) Die Lage der IT-Sicherheit in Deutschland 2009. http://www.bsi.bund.de/ literat/lagebericht/Lagebericht2009.pdf. Abruf am 2009-04-20

Chou T (2008) Seven Software Business Models. Active Book Press

Eder SW (1994) Grüne Computer. WIRTSCHAFTSINFORMATIK 36(6):600-603

Elkington J (1997) Cannibals with forks. The triple bottom line of $21^{\text {st }}$ century business. Captone, Oxford

Freeman RE (1984) Strategic management: A stakeholder approach. Boston

Hauff V (1987) Unsere gemeinsame Zukunft: Der Brundtland-Bericht der Weltkommission für Umwelt und Entwicklung. Eggenkamp, Greven

Koomey JG (2007) Estimating total power consumption by servers in the U.S. and the world, Final report. Stanford University

Krcmar H (2005) Informationsmanagement, 4. Aufl. Springer, Heidelberg

Österle H, Blessing D (2000) Business engineering model. In: Österle H, Winter R (Hrsg) Business Engineering: Auf dem Weg zum Unternehmen des Informationszeitalters. Springer, Heidelberg, $\mathrm{S} 61-80$

Schmidt NH, Erek K, Kolbe LM, Zarnekow R (2009) Towards a procedural model for sustainable information systems management. In: HICSS, $42^{\text {nd }}$ Hawaii international conference on system sciences, S 1-10

Wade M, Hulland J (2004) The resource-based view and information systems research, review, extension, and suggestions for future research. MIS Quarterly 28(1):107-142

\section{Praxisorientierte Einfüh- rung in das Internetrecht}

$\lambda$
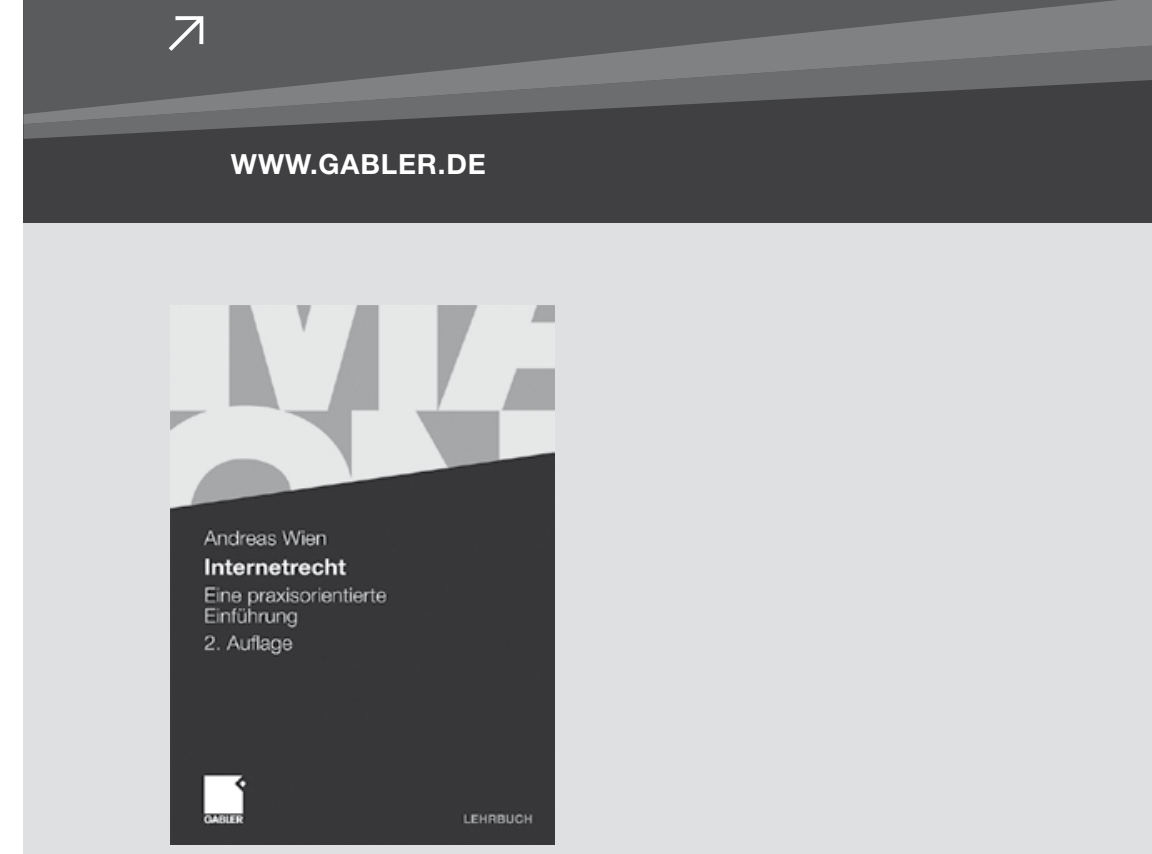

Wien, Andreas

\section{Internetrecht}

Eine praxisorientierte Einführung

2., überarb. Aufl. 2009. XVI, 235 S.

Br. EUR 26,90

ISBN 978-3-8349-1502-3

Das Internet gewinnt in der Wirtschaft und Gesellschaft zunehmend an Bedeutung und ist aus vielen Bereichen nicht mehr wegzudenken. Durch eine Flut von Gesetzen und der in vielen Bereichen des Internetrechts noch nicht gefestigten Rechtsprechung besteht bisweilen große Unsicherheit bei den Nutzern. „Internetrecht“ führt leicht verständlich und zugleich fundiert in alle wichtigen Themen ein. Besonderen Wert legt der Autor auf einen hohen Praxisbezug. Anhand vieler Beispielfälle wird ein konkreter Einblick in die Anwendungsmöglichkeiten und Einsatzfelder gegeben. Alle aktuellen Gesetzesreformen sind berücksichtigt. Die zweite Auflage trägt aktuellen Entwicklungen Rechnung und vertieft insbesondere das Thema „Hacking und Phishing“.

Einfach bestellen: kerstin.kuchta@gwv-fachverlage.de Telefon $+49(0) 611$. 7878-626

KOMPETENZ IN

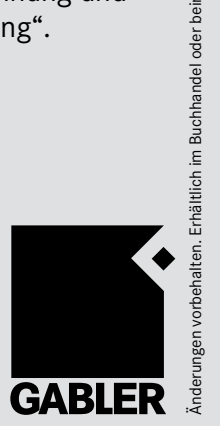

